\title{
IDENTIFICATION OF FERRET ACE2 AND ITS RECEPTOR FUNCTION FOR SARS-CORONAVIRUS
}

\author{
Aya Zamoto, Fumihiro Taguchi, Shuetsu Fukushi, Shigeru Morikawa, and \\ Yasuko K. Yamada*
}

\section{INTRODUCTION}

Severe acute respiratory syndrome associated coronavirus (SARS-CoV) was the causative agent of SARS, which occurred as an emerging pneumonic disease in 2002. ${ }^{1-3}$ The epidemiological investigations showed that several wild animals such as Himalayan palm civet and raccoon dog had been infected by SARS-CoV. ${ }^{4}$ Experimental infection to several laboratory animal species such as mouse, hamster, ferret, cat, and monkey revealed the susceptibility of those animal species to SARS-CoV infection, ${ }^{5-7}$ however, severe clinical manifestations were observed only in the ferret. ${ }^{8}$

Human angiotensin-converting enzyme 2 (ACE2), a metallopeptidase, was demonstrated to be a functional receptor for SARS-CoV. ${ }^{9}$ To see whether ACE2 function as receptor is attributed to the disease manifestation, we cloned a ferret ACE2 (feACE2) gene and compared the feACE2 with that of human or mouse ACE2 in terms of receptor functionality for SARS-CoV.

\section{MATERIALS AND METHODS}

Amplification of partial ACE2 gene by RT-PCR: RNAs were extracted from heart, lung, kidney, and small intestine of a ferret. To select a suitable organ to amplify a full-length ACE2 gene, we amplified a partial gene of ACE2 (952 bp in size) by RT-PCR. As a control, all RNA specimens were subjected to RT-PCR with $B$-actin specific primers.

Determination of nucleotide sequence of ferret ACE2 (feACE2): Two overlapping regions of feACE2 genes were amplified by RT-PCR from kidney RNA. One contained the region 5' to the initiation codon of feACE2 gene, while the other was located 3' to a stop codon. The complete nucleotide sequence of feACE2 gene was determined by direct

* National Institute of Infectious Diseases, Musashimurayama, Tokyo 208-0011, Japan. 
sequencing and was deposited in GenBank under accession number AB208708. Putative signal peptide, zinc binding, transmembrane and $\mathrm{N}$-glycosylation sites were searched by PROSITE. We aligned entire ACE2 sequences of ferret, human (BC039902) and mouse (BC026801), and determined the identities between them by use of Multiple Alignment program in the CLUSTALW.

Expression of feACE2, human ACE2, and mouse ACE2 in HeLa cells: HeLa229 cells were transfected with a recombinant pTarget plasmid (Promega) encoding an entire ACE2 gene using Trans Fast Transfection Reagent (Promega). Transfected cells were selected in the presence of $600 \mu 1 / \mathrm{ml}$ of Geneticin. Clones expressing high levels of ACE2 were selected by immunofluorescence assay (IFA) and Western blot with goat anti-human ACE2 antibody (R\&D Systems).

SARS-CoV replication in ACE2-expressing HeLa229 cells: HeLa cell clones expressing ferret, human, or mouse ACE2 were inoculated with SARS-CoV (Frankfurt-1) at a multiplicity of infection of 0.1 . At 24 hours postinoculation, cells were freeze-thawed and clarified by centrifugation and virus titer was quantified by plaque assay on Vero E6 cells. Standard errors of means were calculated from three experiments.

\section{RESULTS}

The partial feACE2 gene was amplified from RNAs isolated from the lung, heart, kidney, and small intestine by RT-PCR. The amount of amplicon varied among the organs (Fig. 1). An entire feACE2 gene was successfully amplified by RT-PCR from RNA isolated from the kidney. Its sequencing analysis showed that feACE2 consisted of 805 amino acid residues. There were 6 predicted N-glycosylation sites in the feACE2 (Table 1), and 4 of them (aa 53, 322, 546, 690) were conserved between ferret and human ACE2. Amino acid identities of ACE2 between ferret and human or mouse were $82.6 \%$ and $81.5 \%$, respectively (Table 2 ).

We selected 3 HeLa cell clones stably expressing feACE2 by IFA (Fig. 2) and Western blot (data not shown). HeLa cells expressing feACE2 supported SARS-CoV replication to the same extent as those expressing human ACE2 (Fig. 3). Replication of SARS-CoV in feACE2 expressing HeLa cells was over 10 times more efficient than that in mouse ACE2 expressing HeLa cells (Fig. 3).

\section{DISCUSSION AND CONCLUSIONS}

We have determined the complete nucleotide sequence of feACE2 gene and established HeLa cell lines that stably express feACE2. Studies using these cells as well as cells expressing human or mouse ACE2 revealed that feACE2 functions as a SARS-CoV receptor as efficiently as human ACE2, while mouse ACE2 does less efficiently. Our findings shown in this study are in agreement with the observations by $\mathrm{Li}$ et al., who reported that human ACE2 is more efficient than mouse ACE2 in binding to the SARS-CoV S protein. ${ }^{10}$ From these observations, it is postulated that animal species whose ACE2 functions as an efficient SARS-CoV receptor would be a susceptible 
host species for this virus. To establish a good animal model for SARS, a ferret model would be a good candidate. Furthermore, transgenic mouse expressing ferret or human ACE2 may serve a useful animal model for SARS-CoV infection.

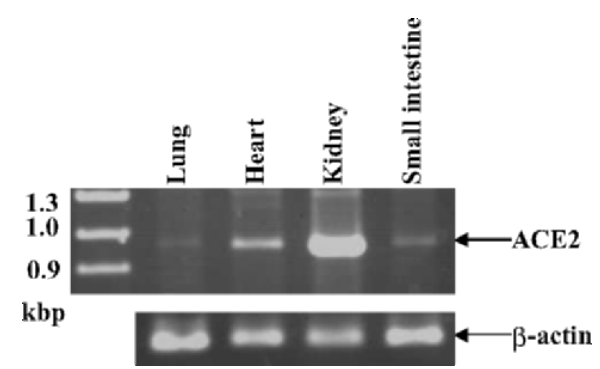

Figure 1. Amplification of ACE2 gene from heart, lung, kidney, and small intestine of ferret by RT-PCR.

Table 1. Predicted positions of motif on ferret ACE2.

\begin{tabular}{ll}
\hline Motif & Position \\
\hline Signal peptide & $1-17$ \\
Zinc binding & $371-381$ \\
Transmembrane & $741-763$ \\
N-glycosylation & 6 sites $(53,299,322,546,660,690)$ \\
\hline
\end{tabular}

Table 2. Identity (\%) of ferret ACE2 in amino acid sequence with human and mouse ACE2.

\begin{tabular}{lccc}
\hline & Human & Ferret & Mouse \\
\hline Human & - & $\mathbf{8 2 . 6} \%$ & $\mathbf{8 2 . 1} \%$ \\
Ferret & & - & $\mathbf{8 1 . 5} \%$ \\
Mouse & & & - \\
\hline
\end{tabular}



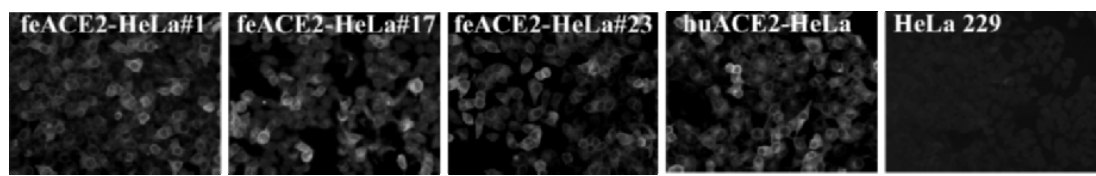

Figure 2. IFA analyses of feACE2 expression in HeLa cell clones.

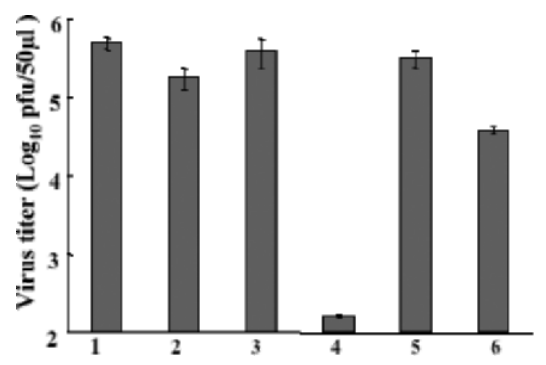

1. PeACE2-HeLa \#1

2. feACE2-HeLa \#17

3. feACE2-HeLa \#23

4. HeLa 229

5. huACE2-HeLa A3

6. MoACE2-HeLa 19-6

Figure 3. Replication of SARS-CoV in HeLa cells expressing ferret ACE2 (lanes 1-3), human ACE2 (lane 5), and mouse ACE2 (lane 6).

\section{REFERENCES}

1. T. G. Ksiazek, D. Erdman, C. S. Goldsmith, et al., A novel coronavirus associated with severe acute respiratory syndrome, N. Engl. J. Med. 348, 1953-1966 (2003).

2. C. Drosten, S. Gunther, W. Preiser, et al., Identification of a novel coronavirus in patients with severe acute respiratory syndrome, N. Engl. J. Med. 348, 1967-1976 (2003).

3. T. Kuiken, R. A. Fouchier, M. Schutten, et al., Newly discovered coronavirus as the primary cause of severe acute respiratory syndrome, Lancet. 362, 263-270 (2003).

4. Y. Guan, B. J. Zheng, Y. Q. He, et al., Isolation and characterization of viruses related to the SARS coronavirus from animals in southern China, Science 302, 276-278 (2003).

5. T. Rowe, G. Gao, R. J. Hogan, et al., Macaque model for severe acute respiratory syndrome, J. Virol. 78, 11401-11404 (2004).

6. A. Roberts, L. Vogel, J. Guarner, et al., Severe acute respiratory syndrome coronavirus infection of golden Syrian hamsters, J. Virol. 79, 503-511 (2005).

7. D. E. Wentworth, L. Gillim-Ross, N. Espina, and K. A. Bernard, Mice susceptible to SARS coronavirus, Emerg. Infect. Dis. 10, 1293-1296 (2004).

8. B. E. Martina, B. L. Haagmans, T. Kuiken, et al., SARS virus infection of cats and ferrets, Nature $\mathbf{4 2 5}, 915$ (2003).

9. W. Li, M. J. Moore, N. Vasilieva, et al., Angiotensin-converting enzyme 2 is a functional receptor for the SARS coronavirus, Nature 426, 450-454 (2003).

10. W. Li, T. C. Greenough, M. J. Moore, et al., Efficient replication of severe acute respiratory syndrome coronavirus in mouse cells is limited by murine angiotensin-converting enzyme 2, J. Virol. 78, 11429-11433 (2004). 